\title{
Traffic-Based Reconfiguration for Logical Topologies in Large-Scale WDM Optical Networks
}

\author{
Yongbing Zhang, Member, IEEE, Masaki Murata, Hideaki Takagi, Fellow, IEEE, and Yusheng Ji, Member, IEEE
}

\begin{abstract}
Wavelength-division multiplexing (WDM) technology has emerged as a promising technology for backbone networks. The optical layer based on WDM technology provides optical routing services to the upper layers such as the packetswitching layer and the time-division multiplexing (TDM) layer over the generalized multiprotocol label-switching (GMPLS) paradigm. The set of all-optical communication channels (lightpaths) in the optical layer defines the logical topology for the upper layer applications. Since the traffic demand of upper layer applications fluctuates from time to time, it is required to reconfigure the underlying logical topology in the optical layer accordingly. However, the reconfiguration for the logical topology is reluctantly disruptive to the network since some lightpaths should be torn down and some traffic has to be buffered or rerouted during the reconfiguration process. Therefore, it needs to have an efficient transition method to shift the current logical topology to the new one so as to minimize the effect of the reconfiguration on the upper layer traffic. This paper proposes several heuristic algorithms that move the current logical topology efficiently to the given target logical topology in large-scale wavelength-routed optical networks. In the proposed algorithms, the performance improvement/degradation of data transmission [transmission delay or distance between a source-destination $(s-d)$ pair] caused by a new lightpath is considered as benefit for establishing the new lightpath. The proposed algorithms construct the new logical topology starting from a lightpath with the largest benefit to the user traffic. Simulation experiments have been performed to evaluate the proposed algorithms in comparison with existing algorithms in a National Science Foundation Network (NSFNET)-like network model with 16 nodes and 25 links. The results show that the proposed algorithms yield much better performance (shorter average packet hot distance) than previous algorithms mostly with comparable computation time.
\end{abstract}

Index Terms-Lightpath, logical topology, multihop connection, optical networks, traffic-based reconfiguration, wavelengthdivision multiplexing (WDM).

\section{INTRODUCTION}

$\mathbf{W}$ AVELENGTH-DIVISION multiplexing (WDM) is a promising technology for using the enormous bandwidth available in an optical communication medium [1], [2]. In a WDM-based network, wavelength multiplexers are utilized to multiplex user signals on a single WDM fiber, and optical

Manuscript received November 23, 2004; revised June 3, 2005. This work was supported in part by the University of Tsukuba under University Research Projects, Research Grant (A), in 2003.

Y. Zhang and H. Takagi are with the Graduate School of Systems and Information Engineering, University of Tsukuba, Tsukuba-shi, Ibaraki 305-8573, Japan (e-mail: ybzhang@sk.tsukuba.ac.jp; takagi@sk.tsukuba.ac.jp).

M. Murata is with the NTT Internet, Inc., Shinjuku-ku, Tokyo 160-0023, Japan.

Y. Ji is with the National Institute of Informatics, Chiyoda-ku, Tokyo 101-8430, Japan (e-mail: kei@nii.ac.jp).

Digital Object Identifier 10.1109/JLT.2005.855663 cross connects (routing nodes) are used to switch the optical signals in optical domain. Routing nodes with a limited number of optical transmitters and receivers (a pair of a transmitter and a receiver is called a transceiver) are interconnected with each other by point-to-point fiber links. A message arriving at one of the input links of a routing node on some wavelength can be switched to any one of the output links on the same wavelength without electronic-optical (E/O) or optical-electronic (O/E) conversion. A route (a set of links) traversed by data between two nodes and formed by an all-optical path on a given wavelength is called a lightpath. The wavelength limitation required for an all-optical transmission path is called the wavelength continuity constraint. It is virtually impossible to realize the whole connections with all lightpaths due to the resource (wavelength, transceiver, etc.) limitations. Therefore, the data transmission from a source to its destination inevitably needs to pass through more than one lightpath and experiences E/O conversion at intermediate lightpath endpoints.

The WDM optical layer in a WDM-based network provides a logical topology comprised of lightpaths to its upper layers such as the packet-switching layer and/or the time-divisionmultiplexing (TDM) layer based on the generalized multiprotocol label-switching (GMPLS) control paradigm [3]-[5]. In the design of a logical topology for a wavelength-routed WDM network, both the physical fiber network and the network traffic pattern of the upper layers should be taken into account. The number of lightpaths along a transmission path from a source to its destination should be limited to as few as possible in order to reduce the delay caused by the $\mathrm{E} / \mathrm{O}$ conversions and the cost of electrical switching at the intermediate lightpath endpoints. Since the traffic pattern in upper layers may fluctuate from time to time, it is vital to reconfigure the logical topology according to the changes in the traffic pattern.

There are two important issues involved in the reconfiguration of a network logical topology [2], [6]-[10]. One issue is how to determine the target logical topology corresponding to the current topology and traffic pattern. The other issue is how to determine a reconfiguration transition sequence shifting the current topology to the new one. Most research until now focuses on the former, but some research focuses on the latter. Furthermore, no research can be found in the literature on realization of the reconfiguration for randomly given new and old logical topologies in large-scale networks. The two issues can also be considered jointly, but a tradeoff should be taken into account between the performance of the target topology and the number of changes to the logical topology [11]. Besides, the topology transition problem still exists even though the number of changes may be reduced using this approach. 
This paper focuses on the reconfiguration transition problem and proposes several heuristic reconfiguration algorithms for large-scale WDM optical networks. The proposed algorithms attempt to move the current logical topology to the given new one while minimizing the disruption to the network. The authors take a lightpath as the minimum unit in the reconfiguration process and try to determine an optimal establishment sequence for the new lightpaths.

Two distinct objectives can be considered for determining a reconfiguration sequence. One objective is from the system viewpoint and is to minimize the disruption of system resources (optical transmitters/receivers) during the reconfiguration operation. Another objective is from the user viewpoint and is to minimize the effect of the reconfiguration on the user traffic. The authors' previous study [12] considered the quantity of disrupted resources as the performance measure and tried to minimize the resource disruption during the reconfiguration process. In this paper, the authors consider the latter objective and take the benefit of establishing a new lightpath to user traffic as the performance measure. The benefit of establishing a new lightpath denotes the improvement/degradation of the transmission delay or the distance from a source to its destination that users can obtain by using the new lightpath. From a user's viewpoint, it is generally more important to choose a new lightpath with a larger benefit than a lightpath with less resource disruption to establish. The proposed algorithms in this paper construct the new logical topology starting from a lightpath with the largest benefit to the user traffic. Those algorithms are evaluated in comparison with the authors' previously proposed algorithms and two extremely simple algorithms by means of simulation experiments.

The rest of the paper is organized as follows. Section II describes the background of this paper and the related work on the logical topology reconfiguration in WDM optical networks. Section III presents the problem formulation and the performance measures used in the paper. Section IV describes the proposed algorithms. Simulation results are shown in Section V, and Section VI summarizes the conclusions.

\section{BACKGROUND AND RELATED WORK}

The logical topology for a WDM-based network should be designed based on both the physical network topology and the traffic pattern of upper layers [2], [7], [8]. The exact solution to this problem can be easily shown NP-hard [13], and, therefore, heuristic approaches are usually used to find realistic solutions. Furthermore, it is vital to reconfigure the logical topology according to the changes in traffic pattern. However, the reconfiguration is disruptive to the network under operation. Therefore, it needs to consider a tradeoff between the performance of the new logical topology and the cost of the topology reconstruction [8], [11], [14]-[17]. Some authors focused on the reconfiguration transition approaches [18]-[21]. However, their models are limited to small networks like local area networks.

Banerjee and Mukherjee [8] have studied the reconfiguration issues for logical topologies in large-scale WDM optical networks. They formulated the reconfiguration problem by using the mixed-integer linear programming (MILP) formulation and proposed a heuristic algorithm to obtain the new logical topology with the minimum cost. They did not mention how to reconfigure the new logical topology. Gencata and Mukherjee [17] proposed an on-line adaptive reconfiguration approach to follow the dynamic changes in traffic patterns without a priori knowledge. Their algorithm reacts promptly to the traffic fluctuation by adding or deleting one lightpath at a time. Sreenath et al. [11] proposed a two-stage approach to the reconfiguration problem. In the first stage, the reconfiguration is limited to a few changes in order to speed up the reconfiguration process and reduce the reconfiguration cost. In the second stage, the topology optimization between consecutive traffic changes is performed in order to make the topology close to the optimal one. The reconfiguration methods described above attempt to limit the differences between the new and the old logical topologies in order to reduce the disruption to the network. However, we still have the problem of how to realize the new logical topology, i.e., how to move the old logical topology to the new one.

Labourdette et al. [18] proposed an efficient reconfiguration method, called the branch exchange, to shift the old topology to the new one in a local area network like a star-coupler configuration. Under their approach, the reconfiguration sequence is determined clearly, and, each time, only one node pair is selected to switch their transmitters and receivers. Kato and Oie [9] proposed several reconfiguration algorithms that move the old logical topology to the new one for a Torus network. However, their model is based on either star or bus physical networks, i.e., there is no wavelength conflict between the new and old lightpaths. Narula-Tam and Modiano [22] and Mohan et al. [23] proposed a reconfiguration method specific to a ring network. Their approach attempts to minimize the disruption to the network and guarantees the connectivity of the network during the reconfiguration process. Bala et al. [24] proposed a hitless reconfiguration approach for the logical topology. They proposed to first establish all the new lightpaths without removing any old lightpaths. The old lightpaths are removed only when the traffic was rerouted through the lightpaths of the new topology. However, in order to realize their approach, additional spare resources (transmitters, receivers, and wavelengths) are needed to establish the new lightpaths without removing the old lightpaths.

Recently, Reddy et al. [25] proposed an approach to realize the reconfiguration for a given set of failure lightpaths in largescale networks. A new lightpath is established for each faulty lightpath with the same starting and ending points. They used the deviation of disrupted time between two faulty lightpaths as their performance measure. In our previous study [12], we considered the topology transition problem from an old topology to its new one, which is determined independently of the old one. We proposed to use a lightpath as the minimum unit to reconfigure the logical topology. We tried to determine a reconfiguration sequence resulting in the minimum disruption to the network resources based on the number of conflict relations between the new and the old lightpaths. However, the performance measures used in this study were restricted only to the utilization of transceivers, and the traffic demand of upper layers was not taken into account. 


\section{PROBlem SPECIFICATION}

In a WDM-based network, each routing node is equipped with add-drop devices and with a limited number of transceivers for data inputs/outputs. A routing node can work as the starting or the ending point of a lightpath at which $\mathrm{E} / \mathrm{O}$ conversions can be performed. Furthermore, a routing node can also work as an intermediate switching point on a lightpath switching an incoming wavelength to the outgoing link using the same wavelength. A lightpath consists of a transmitter at the starting point of the lightpath, a receiver at the ending point, and a wavelength that are assigned to the links along the lightpath from the starting point to the ending point. A logical topology for a WDM-based network is composed of lightpaths that are determined based on the traffic demand of upper layers. The reconfiguration for a logical topology is to realize a given new logical topology based on the current (old) logical topology as shown in Fig. 1. The lightpaths in the new and old logical topologies are denoted by $l_{i}(i=1,2,3,4)$ and $l_{i}^{\prime}(i=1,2, \ldots, 5)$, respectively. The same lightpaths that are used both in the new and old topologies (e.g., $l_{5}^{\prime}$ in the old logical topology and $l_{4}$ in the new logical topology) will remain unchanged. However, the old lightpaths that use any resources, either wavelength, transmitter, or receiver, in conflict with any new lightpath will be reluctantly torn down in order to establish the new lightpath. Since this may cause packet delay or loss, it is crucial to limit the disruption to the network during the reconfiguration process as little as possible.

In this paper, we assume that the average rate of traffic flow from each node to other nodes is given by a traffic matrix. The new and old logical topologies are obtained independently based on the given new and old traffic patterns. We assume that there is no traffic loss and the traffic on the torn-down lightpaths will be rerouted to other paths that are determined using a shortest path algorithm. A newly established lightpath will be available for use once after establishment. The transmission delay (or distance) between a source-destination $(s-d)$ pair is measured by the number of lightpaths the traffic flows from the source to the destination. We take a lightpath as the minimum unit for reconfiguration similar to [12].

To establish a new lightpath having conflict relation with any old lightpath, a two-phase procedure is performed as follows.

1) The old lightpaths that have conflict relations with the new one should be torn down. A control message is forwarded to the nodes along the conflicting old lightpaths, letting them release the required resources.

2) Another control message is forwarded to the nodes along the new lightpath, letting them establish the new lightpath accordingly. Since the time duration for establishing each new lightpath may not vary largely, we assume that the establishment time for any new lightpath is the same. Furthermore, the operations needed to establish a new lightpath is simply denoted by a stage of the whole reconfiguration process. Therefore, we say that there are $n$ stages in the reconfiguration process for a new logical topology with $n$ new lightpaths.

Let $N$ denote the number of nodes in the network. The numbers of transmitters and receivers at node $i$ are denoted

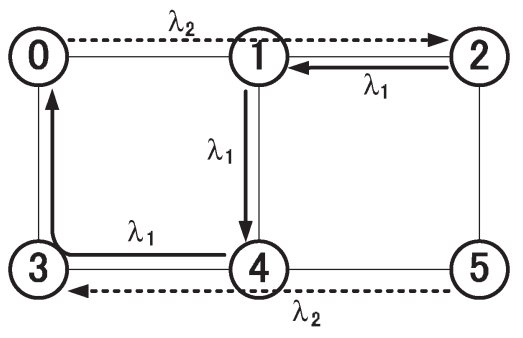

(a)

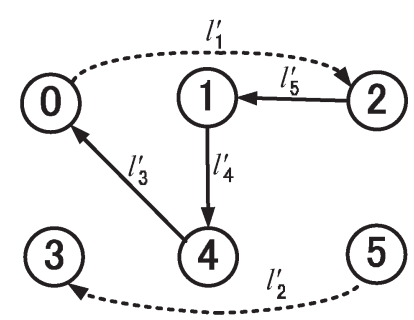

(b)

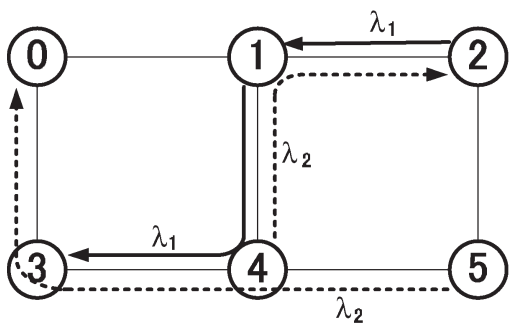

(c)

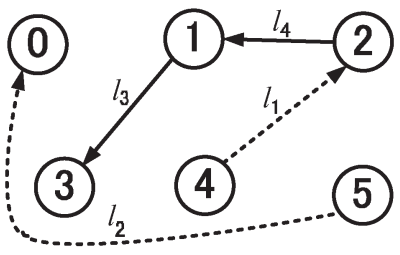

(d)

Fig. 1. Example of the new and old logical topologies. (a) Old lightpaths. (b) Old logical topology. (c) New lightpaths. (d) New logical topology.

by $T_{i}$ and $R_{i}$, respectively. In this paper, it is assumed that $T_{i}=R_{i}, 1 \leq i \leq N$. It is also assumed that each transmitter/ receiver is tunable to any wavelength range. The set of new lightpaths is denoted by $S$. The whole notation used in this paper can be found in the Appendix.

A reconfiguration algorithm needs to realize a reconfiguration process with the minimum disruption to the network and guarantees the quality of service to the upper layers. The following items can be considered as the goals an algorithm needs to achieve.

1) The number of disrupted transceivers at each stage should be minimized. The disrupted transceivers at a stage include the transceivers that are torn down at the stage and those that have been torn down previously.

2) The deviation of the number of disrupted transceivers between different stages should be minimized. A reconfiguration stage with a large number of disrupted transceivers may yield large disruption to the network.

3) The performance degradation of data transmission in the upper layers should be minimized or should not be 
allowed. Ideally, each reconfiguration stage should yield a performance improvement for the data transmission since the resulting topology of each stage approaches the target topology one step closer.

All of these goals may not be realized at the same time, and some tradeoff between them may be needed. The first two goals intend to minimize the resource disruption while the last one intends to minimize the performance degradation of user traffic.

In order to evaluate a reconfiguration algorithm related to the above objectives, we introduce several performance measures. As in our previous research [12], the mean number of disrupted transceivers (MDTs) is defined by the number of disrupted transmitters/receivers on average at each stage during the reconfiguration process and is given by

$$
\operatorname{MDT}=\frac{1}{2|S|} \sum_{i=1}^{|S|} D_{i}
$$

where $|S|$ and $D_{i}(1 \leq i \leq|S|)$ denote the number of new lightpaths and the number of disrupted transceivers at stage $i$, respectively. The above relation can also be written as

$$
\operatorname{MDT}=\frac{1}{2|S|} \sum_{i=1}^{N}\left(\sum_{j=1}^{T_{i}} t_{i j}+\sum_{j=1}^{R_{i}} r_{i j}\right)
$$

where $t_{i j}$ and $r_{i j}$ denote the disrupted time duration (measured in the number of stages) of the $j$ th transmitter and receiver at node $i$, respectively. Note that the disruption time of a transmitter/receiver unused in either the new or the old logical topology is considered to be zero. We can formulate an optimization problem for MDT as

$$
\min \quad \mathrm{MDT}=\frac{1}{2|S|} \sum_{i=1}^{N}\left(\sum_{j=1}^{T_{i}} t_{i j}+\sum_{j=1}^{R_{i}} r_{i j}\right)
$$

subject to $t_{i j}, r_{i j} \geq 0$.

The value of MDT is determined by the establishment order of the new lightpaths, but it is generally difficult to find the best establishment sequence. If the reconfiguration sequence is given, the value of MDT can be calculated as follows. The number of disrupted transceivers at stage $i(1 \leq i \leq|S|)$ can be calculated by

$$
\begin{aligned}
D_{1} & =C_{1} \\
D_{2} & =D_{1}+C_{2}-p_{1} \\
\vdots & \\
D_{i} & =D_{i-1}+C_{i}-p_{i-1} \\
& =\left(C_{1}+C_{2}+\cdots+C_{i}\right)-\left(p_{1}+p_{2}+\cdots+p_{i-1}\right) \\
& =\sum_{j=1}^{i} C_{j}-\sum_{j=1}^{i-1} p_{j}
\end{aligned}
$$

where $C_{i}$ and $p_{i}$ denote, respectively, the numbers of transceivers disrupted and used to establish a new lightpath at stage $i$. We assume that each new lightpath is established at the end instant of a stage. Therefore, we have $p_{j}=2$ for $j=$ $1,2, \ldots,|S|$. Then, MDT is given by

$$
\begin{aligned}
\operatorname{MDT} & =\frac{1}{2|S|} \sum_{i=1}^{|S|} D_{i} \\
& =\frac{1}{2|S|} \sum_{i=1}^{|S|}\left(\sum_{j=1}^{i} C_{j}-\sum_{j=1}^{i-1} p_{j}\right) \\
& =\frac{1}{2|S|}\left(\sum_{i=1}^{|S|} \sum_{j=1}^{i} C_{j}-|S|^{2}+|S|\right) .
\end{aligned}
$$

In order to minimize the deviation of the number of disrupted transceivers between different stages during the reconfiguration process, we introduce a performance measure showing the maximum instantaneous number of disrupted transceivers (MD) as

$$
\mathrm{MD}=\max _{1 \leq k \leq|S|}\{\operatorname{dis}(k)\}
$$

where $\operatorname{dis}(k)$ denotes the instantaneous number of disrupted transceivers at the $k$ th stage. To minimize MD, the following minimization problem can be formulated:

$$
\min \max _{1 \leq k \leq|S|}\{\operatorname{dis}(k)\} .
$$

By establishing each new lightpath $l_{i}$, the traffic between all the $(s-d)$ pairs passing through $l_{i}$ gets some gain $g\left(l_{i}\right)$ because the number of lightpaths between each $(s-d)$ pair may become less than before. The gain indicates the total improvement of the transmission delays (or distances) between the $(s-d)$ pairs passing through $l_{i}$. On the other hand, the traffic passing through the disrupted lightpaths has to be rerouted to other lightpaths. As a result, the transmission delay of rerouted traffic may become longer because the number of lightpaths on the paths of the rerouted traffic may be larger than before. The total delay increment is denoted by the cost $c\left(l_{i}\right)$ for establishing new lightpath $l_{i}$. The difference of the gain and the cost is defined by the benefit of establishing new lightpath $l_{i}$, denoted by $B\left(l_{i}\right)$, and given by

$$
B\left(l_{i}\right)=g\left(l_{i}\right)-c\left(l_{i}\right) .
$$

Each new lightpath may yield a different benefit to the user traffic, and, furthermore, the same new lightpath may yield a different benefit if the reconfiguration sequence differs. It is generally difficult to determine the best establishment sequence for the new lightpaths to produce the largest total benefit since we have to check $|S|$ ! combinations. A heuristic approach can be considered to solve this problem, wherein a new lightpath with the largest benefit at each stage will be established first. For this purpose, we need to find a new lightpath $l_{i}$ to establish that

$$
\max _{l_{i} \in S} B\left(l_{i}\right) .
$$


TABLE I

CONFLICTING NEW AND OLD LIGHTPATHS

\begin{tabular}{c|c|c||c|c|c}
\hline old lightpath & path & wavelength & new lightpath & path & wavelength \\
\hline$l_{1}^{\prime}$ & $0 \rightarrow 1 \rightarrow 2$ & $\lambda_{2}$ & $l_{1}$ & $4 \rightarrow 1 \rightarrow 2$ & $\lambda_{2}$ \\
$l_{2}^{\prime}$ & $5 \rightarrow 4 \rightarrow 3$ & $\lambda_{2}$ & $l_{2}$ & $5 \rightarrow 4 \rightarrow 3 \rightarrow 0$ & $\lambda_{2}$ \\
$l_{3}^{\prime}$ & $4 \rightarrow 3 \rightarrow 0$ & $\lambda_{1}$ & $l_{3}$ & $1 \rightarrow 4 \rightarrow 3$ & $\lambda_{1}$ \\
$l_{4}^{\prime}$ & $1 \rightarrow 4$ & $\lambda_{1}$ & & & \\
\hline
\end{tabular}

Two performance measures as in [8] and [11] are used in this paper to evaluate the effectiveness of a reconfiguration algorithm. One is the weighted packet hop distance and the other is the average packet hop distance.

The weighted packet hop distance for a packet transmitted between an $(s-d)$ pair is defined by the product of the amount of traffic between the $(s-d)$ pair and the number of lightpaths (lightpath hops) in the traffic passing through. Let $x$ and $X$ denote an $(s-d)$ pair and the set of all $(s-d)$ pairs, respectively. For the sake of simplicity, we also use $x$ to denote a path between an $(s-d)$ pair. By letting $T$ denote a certain logical topology, the weighted packet hop distance of an $(s-d)$ pair under topology $T$, denoted by $W_{T}(x)$, is given as

$$
W_{T}(x)=\Lambda(x) H_{T}(x)
$$

where $\Lambda(x)$ and $H_{T}(x)$ denote the amount of traffic of an $(s-d)$ pair and the number of lightpaths along the path between the $(s-d)$ pair, respectively. We assume that there is only one path between an $(s-d)$ pair, and each path is a shortest path, i.e., the path is composed of the smallest number of lightpaths. The average packet hop distance $\alpha(T)$ under topology $T$ is defined by the average number of lightpaths that a packet traverses from source $s$ to destination $d$ and is given by

$$
\begin{aligned}
\alpha(T) & =\frac{1}{\sum_{x \in X} \Lambda(x)} \sum_{x \in X} \Lambda(x) H_{T}(x) \\
& =\frac{1}{\sum_{x \in X} \Lambda(x)} \sum_{x \in X} W_{T}(x) .
\end{aligned}
$$

\section{Proposed Algorithms}

In this paper, we propose to use heuristic algorithms that attempt to minimize the average packet hop distance during the reconfiguration process. That is, at each stage of the reconfiguration process, a new lightpath yielding the minimum average packet hot distance is selected to establish. For this purpose, an auxiliary graph is introduced to show clearly the conflict relations between the new and old lightpaths. In the following subsections, we first describe how to construct the auxiliary graph and then the proposed algorithms in details.

\section{A. Auxiliary Graph}

In the proposed algorithms, the lightpaths in the new logical topology that have no conflict relations with any lightpath in the old logical topology will not be considered. For the conflicting new and old lightpaths, an undirected bipartite auxiliary graph $G_{a}\left(V_{a}, E_{a}\right)$ is introduced, where $V_{a}$ and $E_{a}$ denote the sets

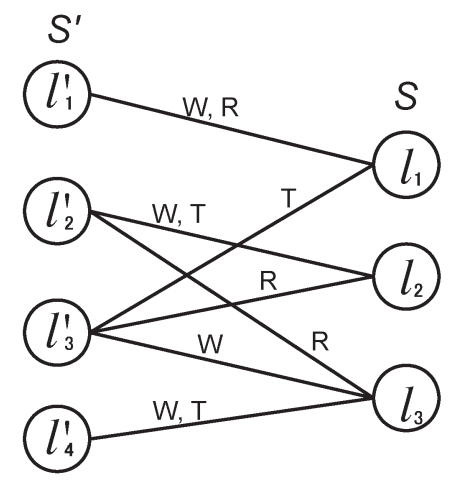

Fig. 2. Auxiliary graph.

of vertices and edges, respectively. The vertices denote the new and old lightpaths that have conflict relations, i.e., $V_{a}=$ $S \cup S^{\prime}$, and the edges denote the specific conflict relations between the new and old lightpaths, i.e., $E_{a}=\left\{\left(l_{i}, l_{j}^{\prime}\right) \mid\right.$ if $l_{i} \in S$ is in conflict with $\left.l_{j}^{\prime} \in S^{\prime}\right\}$.

Note that the conflict relationship may come from the conflicts of wavelength, transmitter, and/or receiver. For example, for the new and old logical topologies shown in Fig. 1, we have the conflicting new and old lightpaths as shown in Table I. Assuming that each node has one transceiver and each link has two wavelengths, the auxiliary graph for Table I is created as shown in Fig. 2. The conflict relations between the new and old lightpaths due to wavelength, transmitter, and receiver are indicated by $W, T$, and $R$ on edges, respectively, as shown in Fig. 2. Generally, the auxiliary graph may consist of multiple disjoint components each of which is independent of others. That is, each new lightpath in a component has conflict relations only with the old lightpaths in the same component and can be established without interference of any lightpath in other components. The algorithms proposed in this paper focus on only one component, and they can be used recursively to solve the whole reconfiguration problem.

\section{B. Fixed Most-Benefit-First Algorithm}

Intuitively, a new lightpath leading to a greater benefit to the reconfiguration should be established earlier. In Fixed Most-Benefit-First (Fix-MBF), the benefit of establishing a new lightpath is determined by using the initial auxiliary graph and the initial traffic flow pattern. The gain obtained by establishing a new lightpath is defined by the reduction quantity in the weighted packet hop distance experienced by the traffic passing through the new lightpath. On the other hand, the cost for establishing a new lightpath is defined by the incremental delay experienced by the rerouted traffic. 
The gain function for establishing new lightpath $l_{i} \in S$, denoted by $g_{\text {fix }}\left(l_{i}\right)$, equals the difference of the weighted packet hop distance values in the old and new logical topologies before and after establishing lightpath $l_{i}$. Let $X^{\prime}$ denote the set of $(s-d)$ pairs such that a path $x \in X^{\prime}$ passes through lightpath $l_{i}$. Furthermore, let $T_{O}\left(l_{i}\right)$ denote the logical topology in which only new lightpath $l_{i}$ is established over the old logical topology $T_{O}$. Then, we have

$$
g_{\text {fix }}\left(l_{i}\right)=\sum_{x \in X^{\prime}}\left(W_{T_{O}}(x)-W_{T_{O}\left(l_{i}\right)}(x)\right)
$$

Conversely, the cost function $c_{\text {fix }}\left(l_{i}\right)$ for establishing new lightpath $l_{i} \in S$ equals the total incremental delay in the weighted packet hop distance caused by traffic rerouting. Let $X^{\prime \prime}$ denote the set of $(s-d)$ pairs such that a path $x \in X^{\prime \prime}$ passes through old lightpath $l_{i}^{\prime} \in S^{\prime}$ in conflict relation with $l_{i}$. Then, the cost function is given by

$$
c_{\mathrm{fix}}\left(l_{i}\right)=\sum_{x \in X^{\prime \prime}}\left(W_{T_{O}\left(l_{i}\right)}(x)-W_{T_{O}}(x)\right) .
$$

Hence, the benefit of establishing new lightpath $l_{i}$, denoted by $B_{\text {fix }}\left(l_{i}\right)$, is given by

$$
B_{\mathrm{fix}}\left(l_{i}\right)=g_{\mathrm{fix}}\left(l_{i}\right)-c_{\mathrm{fix}}\left(l_{i}\right) .
$$

In Fix-MBF, we first calculate the gain $g_{\text {fix }}\left(l_{i}\right)$ and the cost $c_{\text {fix }}\left(l_{i}\right)$ for each new lightpath $l_{i} \in S$. Then, we choose the lightpath with the greatest benefit to establish. The Fix-MBF algorithm has the following five steps.

Step 1) Create the auxiliary graph $G_{a}\left(V_{a}, E_{a}\right)$ and let $T=T_{O}$.

Step 2) Calculate the benefit of establishing each new lightpath using (8).

Step 3) Determine lightpath $\ell$ with the greatest benefit in $S$ such that

$$
\ell=\arg \max _{l_{i} \in S} B_{\mathrm{fix}}\left(l_{i}\right)
$$

Step 4) Set up lightpath $\ell$, and update $T$ and $G_{a}\left(V_{a}, E_{a}\right)$ as

$$
\begin{aligned}
T & =T+\{\ell\}-N(\ell) \\
S & =S \backslash\{\ell\} \\
S^{\prime} & =S^{\prime} \backslash N(\ell)
\end{aligned}
$$

where $N(\ell)$ denotes the set of old lightpaths that have conflict relations with lightpath $\ell$.

Step 5) If $S=\emptyset$, let $T_{N}=T$ and stop. Otherwise, go to Step 3).

\section{Adaptive Most-Benefit-First Algorithm}

The logical topology of a network along with the traffic pattern evolves gradually toward the target logical topology as the reconfiguration process proceeds. Consequently, the gain and the cost of the remaining unestablished lightpaths may change accordingly. Therefore, it is preferable to select dynamically the best new lightpath to establish. The Adaptive Most-Benefit-First (Ad-MBF) algorithm takes into account the dynamical changes of the logical topology. The weighted packet hop distance for each $(s-d)$ pair is updated at each stage of the reconfiguration process. For a logical topology $T$ during the reconfiguration process and a new lightpath $l_{i} \in S$ in Ad-MBF, the benefit of establishing new lightpath $l_{i}$ is defined by

$$
B_{\mathrm{ad}}\left(l_{i}\right)=g_{\mathrm{ad}}\left(l_{i}\right)-c_{\mathrm{ad}}\left(l_{i}\right)
$$

where

$$
\begin{aligned}
& g_{\text {ad }}\left(l_{i}\right)=\sum_{x \in X^{\prime}}\left(W_{T}(x)-W_{T\left(l_{i}\right)}(x)\right) \\
& c_{\text {ad }}\left(l_{i}\right)=\sum_{x \in X^{\prime \prime}}\left(W_{T\left(l_{i}\right)}(x)-W_{T}(x)\right) .
\end{aligned}
$$

The Ad-MBF algorithm consists of the following five steps.

Step 1) Create the auxiliary graph $G_{a}\left(V_{a}, E_{a}\right)$ and let $T=T_{O}$.

Step 2) Calculate/recalculate the benefit $B_{\text {ad }}\left(l_{i}\right)$ of establishing each new lightpath $l_{i} \in S$ using (9).

Step 3) Determine lightpath $\ell$ with the greatest benefit in $S$ such that

$$
\ell=\arg \max _{l_{i} \in S} B_{\mathrm{ad}}\left(l_{i}\right)
$$

Step 4) Set up lightpath $\ell$, and update $T$ and $G_{a}\left(V_{a}, E_{a}\right)$ as

$$
\begin{aligned}
T & =T+\{\ell\}-N(\ell) \\
S & =S \backslash\{\ell\} \\
S^{\prime} & =S^{\prime} \backslash N(\ell) .
\end{aligned}
$$

Step 5) If $S=\emptyset$, let $T_{N}=T$ and stop. Otherwise, go to Step 2).

\section{Minimal Average Packet Hop Distance Lightpath First Algorithm}

In Ad-MBF, the update of the logical topology is only considered locally to the traffic passing through the newly established lightpath and its conflicting old lightpaths. However, each new lightpath may have wider impact on the overall system performance, i.e., the average packet hop distance. The Minimal Average Packet Hop Distance Lightpath First (MAPF) algorithm recalculates the average packet hop distance for each unestablished new lightpath at each stage, and a new lightpath leading to the minimum average packet hop distance will be selected to establish. For this purpose, the benefit of establishing a new lightpath $l_{i}$ under logical topology $T$ is defined by the negative average packet hop distance at the next stage, i.e., the value of the average packet hop distance after establishing lightpath $l_{i}$

$$
B_{\text {ave }}\left(l_{i}\right)=-\alpha\left(T\left(l_{i}\right)+\left\{l_{i}\right\}-N\left(l_{i}\right)\right) .
$$

Since the MAPF algorithm recalculates the paths for all the $(s-d)$ pairs, its computational complexity should be inevitably 


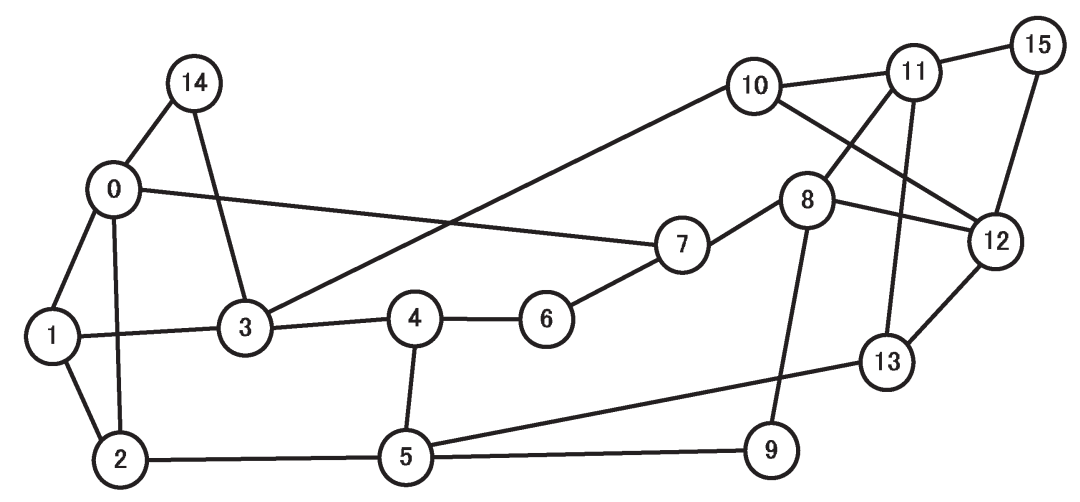

Fig. 3. NSFNET-like network model.

higher than the previous two algorithms. The MAPF algorithm has five steps as follows.

Step 1) Create the auxiliary graph $G_{a}\left(V_{a}, E_{a}\right)$ and let $T=T_{O}$.

Step 2) Calculate/recalculate the benefit $B_{\text {ave }}\left(l_{i}\right)$ of establishing each new lightpath $l_{i} \in S$ using (10).

Step 3) Determine lightpath $\ell$ with the greatest benefit in $S$ such that

$$
\ell=\arg \max _{l_{i} \in S} B_{\text {ave }}\left(l_{i}\right) .
$$

Step 4) Set up lightpath $\ell$, and update $T$ and $G_{a}\left(V_{a}, E_{a}\right)$ as

$$
\begin{aligned}
T & =T+\{\ell\}-N(\ell) \\
S & =S \backslash\{\ell\} \\
S^{\prime} & =S^{\prime} \backslash N(\ell) .
\end{aligned}
$$

Step 5) If $S=\emptyset$, let $T_{N}=T$ and stop. Otherwise, go to Step 2).

\section{Simulation EXPERIMENTS}

Simulation experiments have been conducted to evaluate the proposed algorithms in comparison with existing algorithms. The network model used in the experiments is a National Science Foundation Network (NSFNET)-like network with 16 nodes and 25 links shown in Fig. 3. The traffic rates between node pairs for both the new and old logical topologies are randomly created according to two different traffic types as in [8]: One is uniformly distributed over the range of $[0, \Gamma C]$ with probability $p(0 \leq p \leq 1)$, and the other is over the range of $[0, C]$ with probability $(1-p)$, where $\Gamma$ and $C$ are given constants. In the experiments, the traffic demand from node $i$ to node $j$ is distinguished from the demand from node $j$ to node $i$. Therefore, the transmission path for the connection from node $i$ to node $j$ is independent of that from node $j$ to node $i$. The logical topology for a given traffic pattern is determined by using the max multihop (MM) algorithm proposed in [2]. The new logical topology is determined based solely on the given traffic pattern and is independent of the old logical topology. This is because of the fact that we expect to evaluate the efficiency of the proposed algorithms when there are a large number of lightpaths needing to reconfigure. In reality, the new
TABLE II

COMPUTATION Times (MS) OF THE Algorithms

\begin{tabular}{r|r|rrrrrr}
\hline T/R/W & $|S|$ & LPF & SPF & MDPF & Fix-MBF & Ad-MBF & MAPF \\
\hline 2 & 26 & 3.5 & 3.5 & 3.7 & 12.3 & 100.2 & 83.0 \\
4 & 55 & 7.1 & 7.3 & 8.1 & 27.3 & 388.4 & 345.2 \\
5 & 69 & 8.8 & 9.1 & 10.5 & 34.9 & 587.0 & 545.5 \\
8 & 113 & 15.4 & 15.3 & 21.3 & 61.6 & 1428.8 & 1467.7 \\
10 & 141 & 20.0 & 19.7 & 30.9 & 84.1 & 2233.9 & 2407.4 \\
\hline
\end{tabular}

logical topology should be designed by taking the old logical topology into consideration.

It is assumed that each node has wavelength-switching functionality. It is also assumed that each node in the network has the same number of transceivers and each link has the same number of wavelengths. In the experiments, we consider the case in which the number of transceivers at a node and the number of wavelengths at a link are all the same, and they are denoted by a symbol " $T / R / W$." We consider two kinds of traffic patterns. In one traffic pattern, the flow rates between node pairs are almost uniformly distributed, while in another traffic pattern, the flow rates are unevenly distributed. The parameter $\Gamma$ is used to determine the traffic imbalance. When $\Gamma=1$, the traffic is uniformly balanced, but when $\Gamma$ becomes larger, the traffic imbalance becomes larger. The parameter settings used in the experiments are as follows: $p=0.3$; $C=1$; and $\Gamma$ is set to 2 and 10 , respectively. The parameter $T / R / W$ is examined with the values one by one from 2 to 10. The simulation program has been developed using JAVA and executed on a LINUX server with two $2.8-\mathrm{GHz}$ central processing units (CPUs).

In order to compare our algorithms with existing algorithms, we implement the following three algorithms proposed in [12]: 1) the longest lightpath first (LPF) algorithm; 2) the shortest lightpath first (SPF) algorithm; and 3) the minimal disrupted lightpath first (MDPF) algorithm. The LPF and SPF algorithms are extremely simple and are used for comparison with others. The LPF algorithm constructs the new lightpaths starting with the longest one and continuing to the shorter ones according to the number of hops of the lightpaths in the physical network. On the other hand, the SPF algorithm constructs the new lightpaths conversely starting with the shortest one. In MDPF, the new lightpath with the minimal number of conflicting old lightpaths is established at each stage of the reconfiguration procedure. Interested readers can refer to [12] for further details in the implementation of these algorithms. The performance 


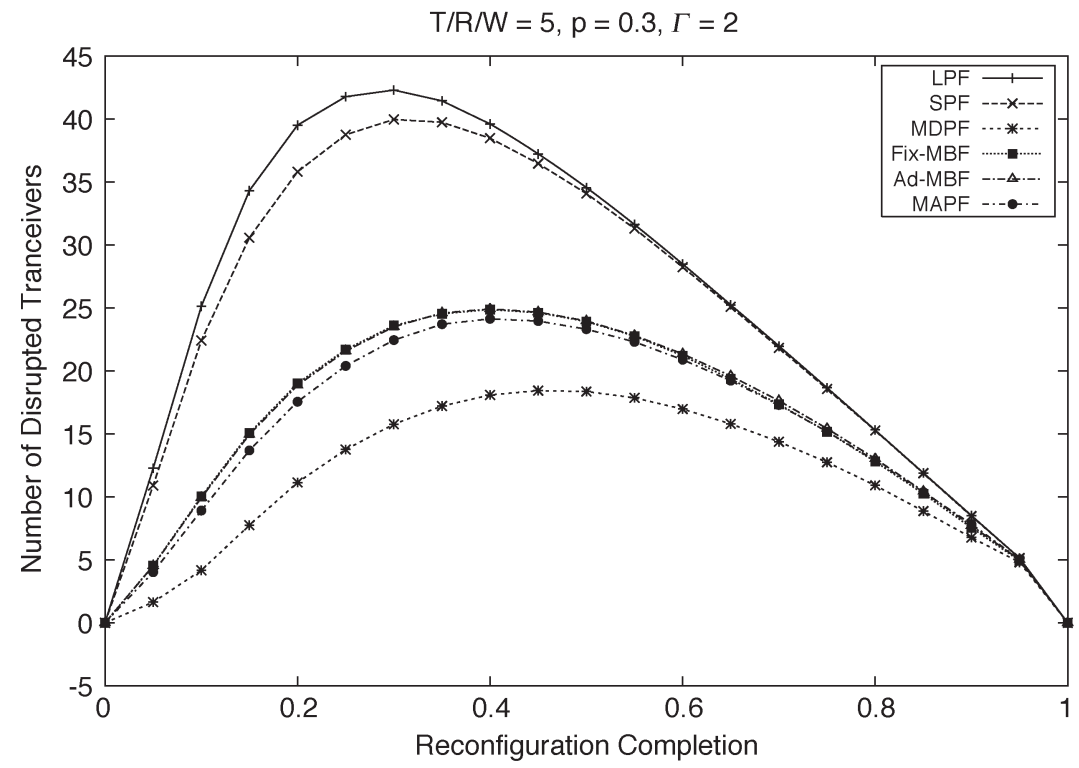

(a)

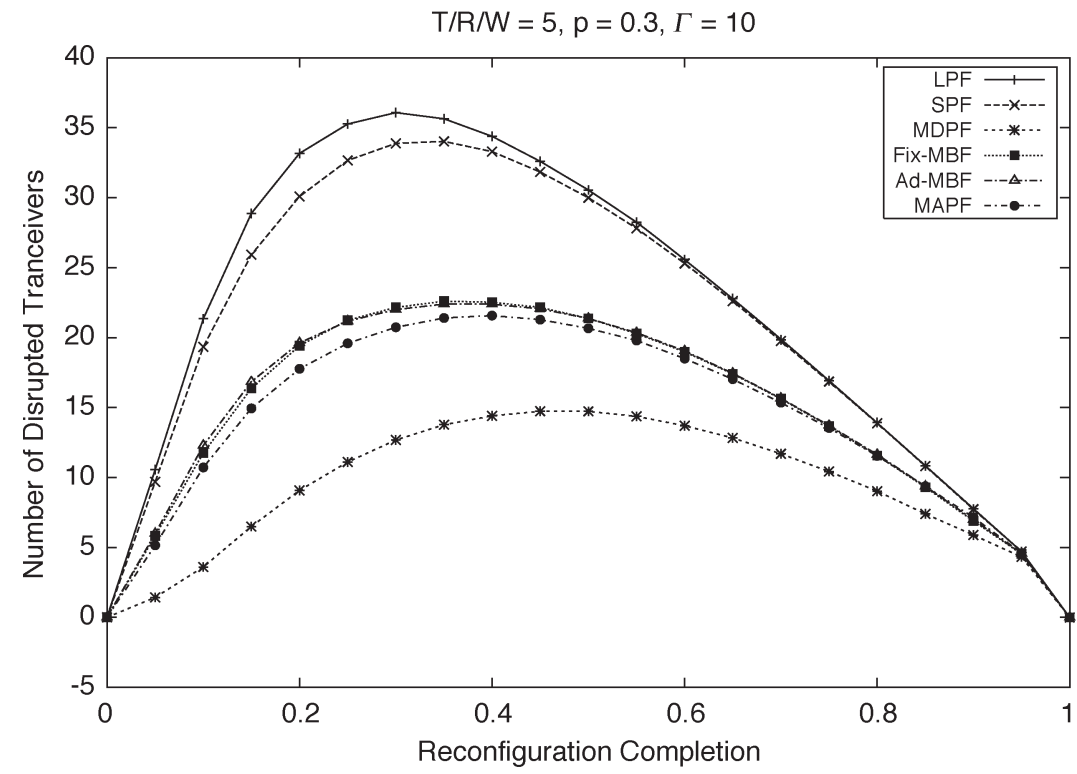

(b)

Fig. 4. Number of disrupted transceivers for $T / R / W=5$ when (a) $p=0.3, \Gamma=2$, and (b) $p=0.3, \Gamma=10$.

measures used for evaluation are, as described in Section II, the number of disrupted transceivers, the maximum number of disrupted transceivers during the reconfiguration process, and the average packet hop distance. The computation times for executing the algorithms under consideration are also examined.

\section{A. Computational Time}

Table II shows the number of new lightpaths $|S|$ having conflict relations with the old lightpaths, and the computation times of the algorithms under consideration for various values of $T / R / W$ when $p=0.3$ and $\Gamma=10$. The results shown in Table II are the average values obtained from 500 simulation runs, each of which has been executed with a distinct traffic pattern.
It can be seen that the number of new lightpaths $|S|$ and the computation times of the algorithms except Ad-MBF and MAPF increase proportionally to the value of $T / R / W$. It is observed that the computation time of LPF, SPF, or MDPF is shorter than either of Fix-MBF, Ad-MBF, or MAPF. Although the computation times of Ad-MBF and MAPF are longer than others in all cases and grow exponentially, they still lie in the practical domain with a reasonable problem size. For example, when $T / R / W=10$ (there are more than 140 new lightpaths needing to establish), the MAPF algorithm takes only around $2.5 \mathrm{~s}$ for the whole reconfiguration process. Note that the difference between the new and old logical topologies can be controlled to be small enough in practice, and, therefore, the computation time for reconfiguration should not be a key factor. Besides, the most important issue for reconfiguration is how to limit the disruption to the network 


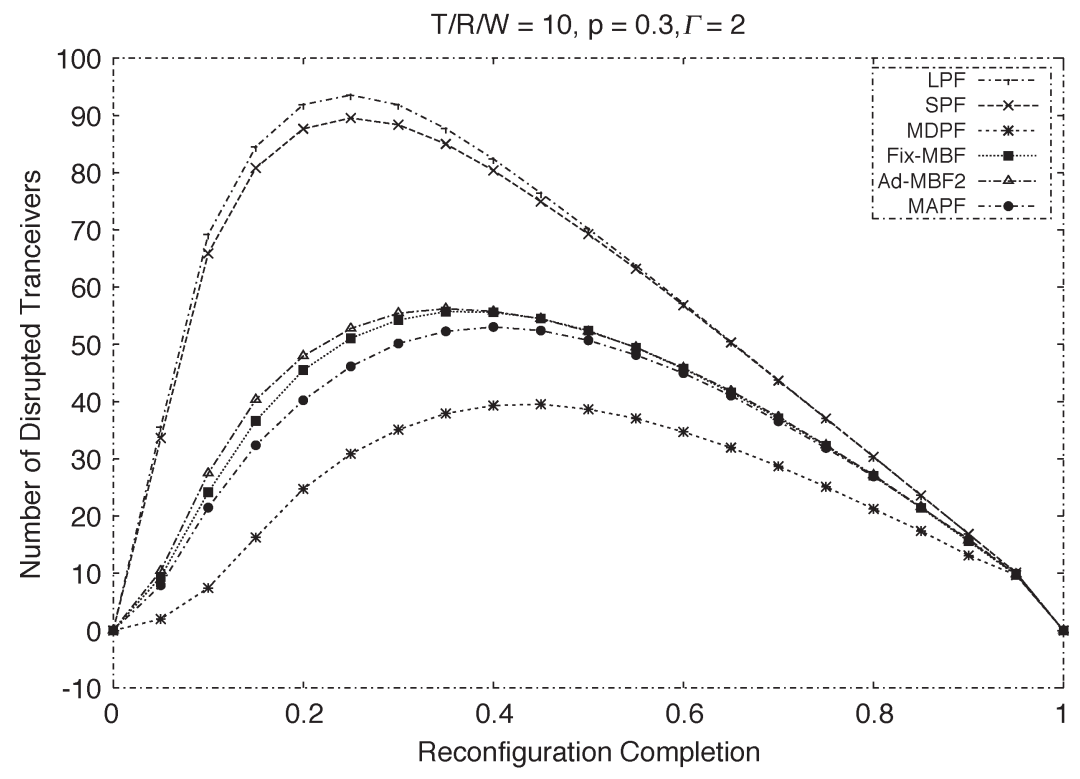

(a)

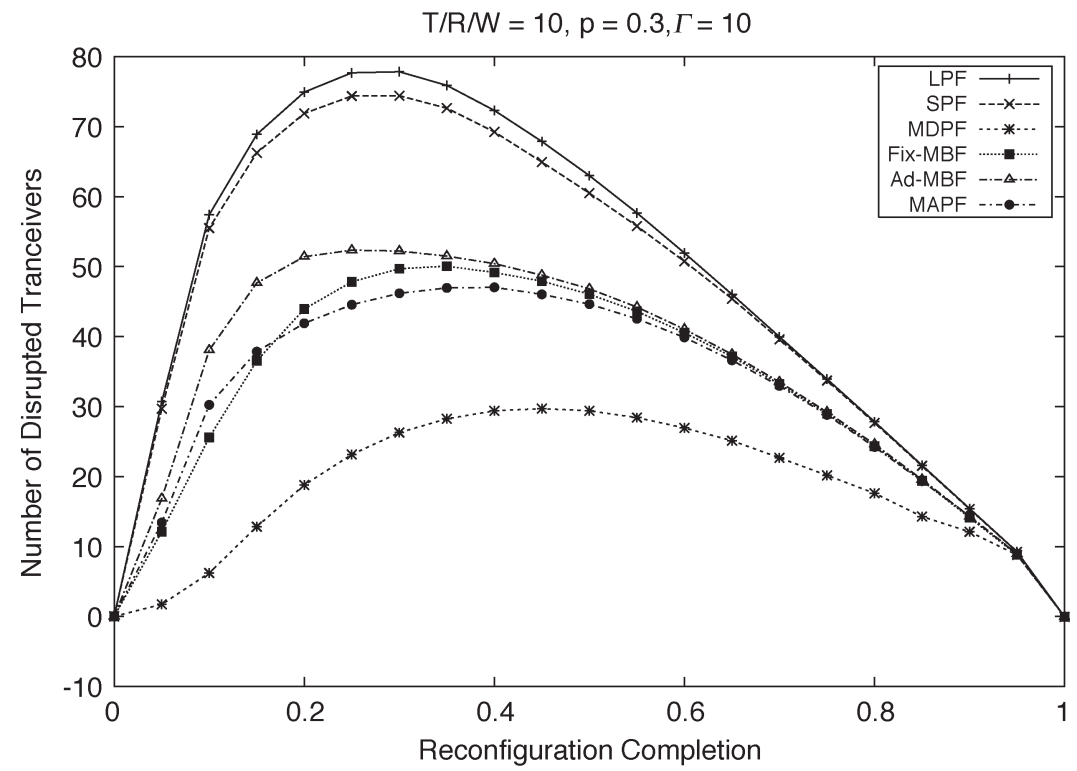

(b)

Fig. 5. Number of disrupted transceivers for $T / R / W=10$ when (a) $p=0.3, \Gamma=2$, and (b) $p=0.3, \Gamma=10$.

other than the reconfiguration time so that upper layer applications will not perceive the reconfiguration operation.

\section{B. Comparison of Performance}

We next evaluate the performance of our proposed algorithms. Since the number of new lightpaths (reconfiguration stages) for each distinct traffic pattern may be different even for the same value of $T / R / W$, we normalize the number of reconfiguration stages to 1 . Therefore, the $x$ axis of the graphs represents the reconfiguration completion in percentage. The results shown in the figures are the mean values obtained from 500 simulation runs with $95 \%$ confidence intervals. The confidence intervals are obtained within $3 \%$ of the sample means and are not shown in the figures.
Figs. 4 and 5 show the numbers of disrupted transceivers of the algorithms under consideration for the cases where the values of $T / R / W$ are 5 and 10 , respectively. From these figures, it can be seen that the algorithms show similar behaviors for various values of $T / R / W$ and traffic patterns. The MDPF algorithm yields the best performance as expected, and the LPF and SPF algorithms show the worst. By comparing Fig. 4(a) (where $\Gamma=2$ ) with Fig. 4(b) (where $\Gamma=10$ ), it is observed that the difference in the number of disrupted transceivers between MDPF and the proposed algorithms increases slightly when the value of $T / R / W$ becomes larger.

Fig. 6(a) and (b) shows the values of MDT and MD, respectively, for various values of $T / R / W$. From these two figures, it is observed that MDT and MD of each algorithm increases proportionally to the value of $T / R / W$. It is also observed 


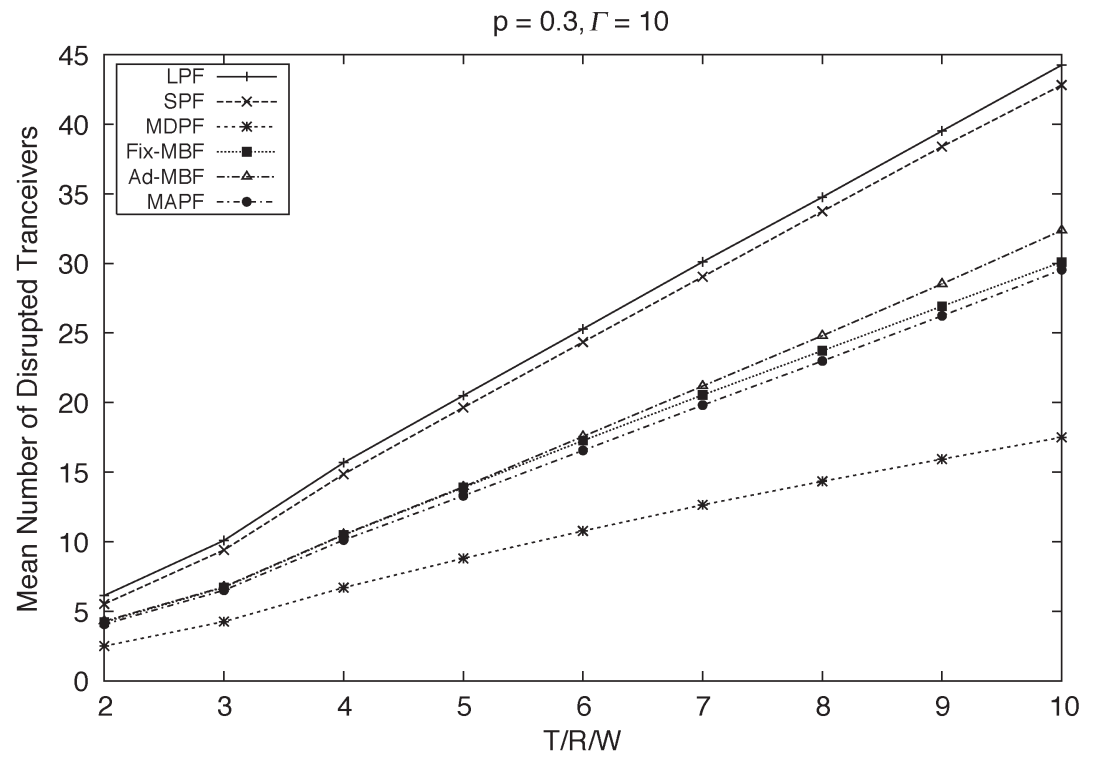

(a)

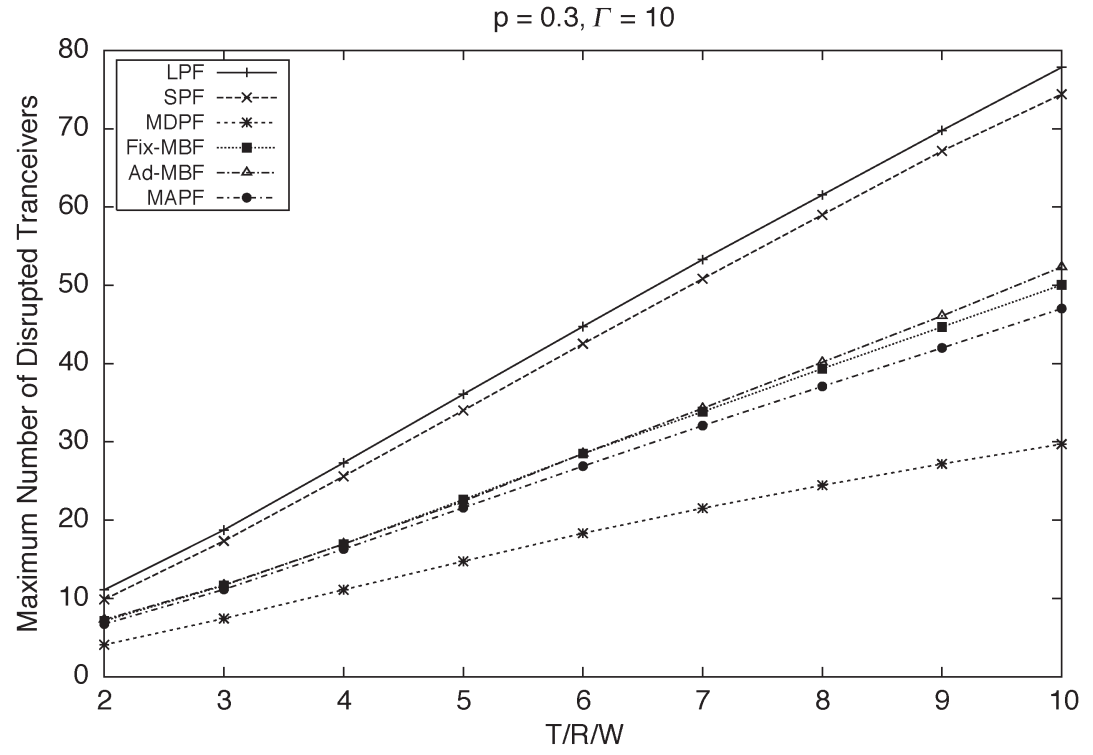

(b)

Fig. 6. Number of disrupted transceivers for various $T / R / W$ when $p=0.3$ and $\Gamma=10$. (a) Mean number of disrupted transceivers (MDT). (b) Maximum number of disrupted transceivers (MD).

that the difference of the maximum number of disrupted transceivers between MDPF and the proposed algorithms increases linearly when the value of $T / R / W$ becomes larger.

Figs. 7 and 8 plot the performance measure $\alpha(T)$ for the algorithms under consideration. It can be seen that the performance of LPF or SPF is far worse than the others. From these figures, it is observed that the average packet hop distance $\alpha(T)$ of each proposed algorithm is better than that of MDPF in most cases, and the advantage becomes significant when the traffic load is uneven or the value of $T / R / W$ becomes large. It is also observed that the proposed algorithms perform not only better than MDPF but, more important, they also provide a better value of $\alpha(T)$ than the initial value of $\alpha(T)$ before reconfiguration at each stage, especially when the traffic imbalance is significant (e.g., when $\Gamma=10$ ) and the value of $T / R / W$ is large (e.g., when $T / R / W=10$ ). This means that an upper layer user can usually sense the improvement rather than the degradation in performance during the reconfiguration process.

The values of $\alpha(T)$ of Ad-MBF and MAPF are improved obviously within several stages from the beginning of the reconfiguration process. This result becomes obvious when the traffic load is uneven, say, when $\Gamma=10$ as shown in Figs. 7(b) and 8(b). However, the worst value of $\alpha(T)$ in MDPF is constantly worst than the initial average packet hop distance with various parameter settings. This is because of the fact that MDPF does not take into account of the upper layer traffic in reconfiguration decisions. It is observed that the largest performance improvement in the value of $\alpha(T)$ of MAPF over MDPF is more than $7 \%$ when $\Gamma=10$ and $T / R / W=5$ as shown in Fig. 7(b), and the improvement becomes near $10 \%$ when $\Gamma=10$ and $T / R / W=10$ as shown in Fig. 8(b). 


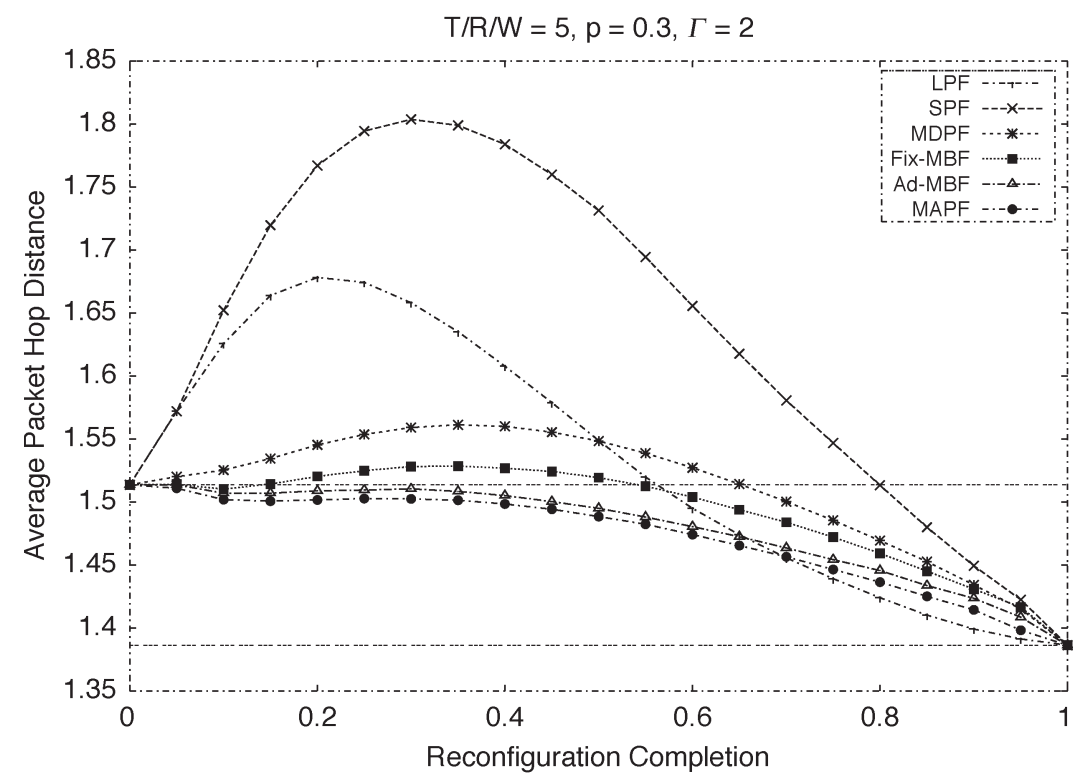

(a)

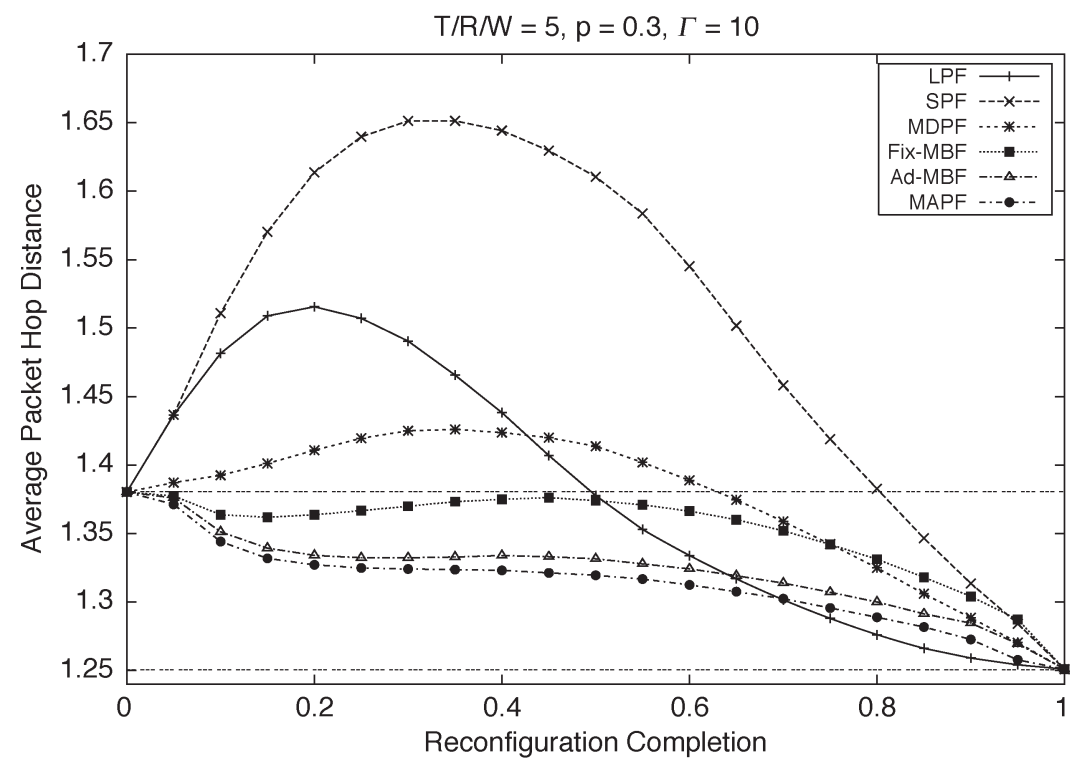

(b)

Fig. 7. Average packet hop distance for $T / R / W=5$ when (a) $p=0.3, \Gamma=2$, and (b) $p=0.3, \Gamma=10$.

The advantages of the proposed algorithms, especially MAPF, over the previous algorithms can be summarized as follows. The performance of data transmission in the proposed algorithms becomes better than the initial performance value during the whole reconfiguration process and the performance improvement is achieved mostly within several stages from the beginning of the reconfiguration operation. This indicates that the upper layer users can obtain the benefit of reconfiguration very fast using the proposed algorithms. On the other hand, the previous algorithms will suffer since the performance of data transmission is degraded during the reconfiguration process. Upper layer users may, therefore, feel uneasy because they have to experience the performance degradation during the reconfiguration process. Furthermore, when the traffic load becomes uneven and the value of $T / R / W$ is large, the advantage of the proposed algorithms over previous algorithms in terms of data transmission performance (average packet hop distance) becomes significant. Since the network traffic in real systems may be highly unbalanced, the proposed algorithms may yield much better performance.

\section{CONCLUSION}

In this paper, we proposed three reconfiguration algorithms, fixed most-benefit-first (Fix-MBF), adaptive most-benefit-first (Ad-MBF), and minimal average packet hop distance lightpath first (MAPF), that take into account of the traffic demand of upper layers. These algorithms are evaluated by using two kinds of performance measures: one indicating the quantity of disrupted resources, i.e., the mean and the maximum numbers of disrupted transceivers, MDT and MD, and the other indicating the impact on the user performance, i.e., the average 


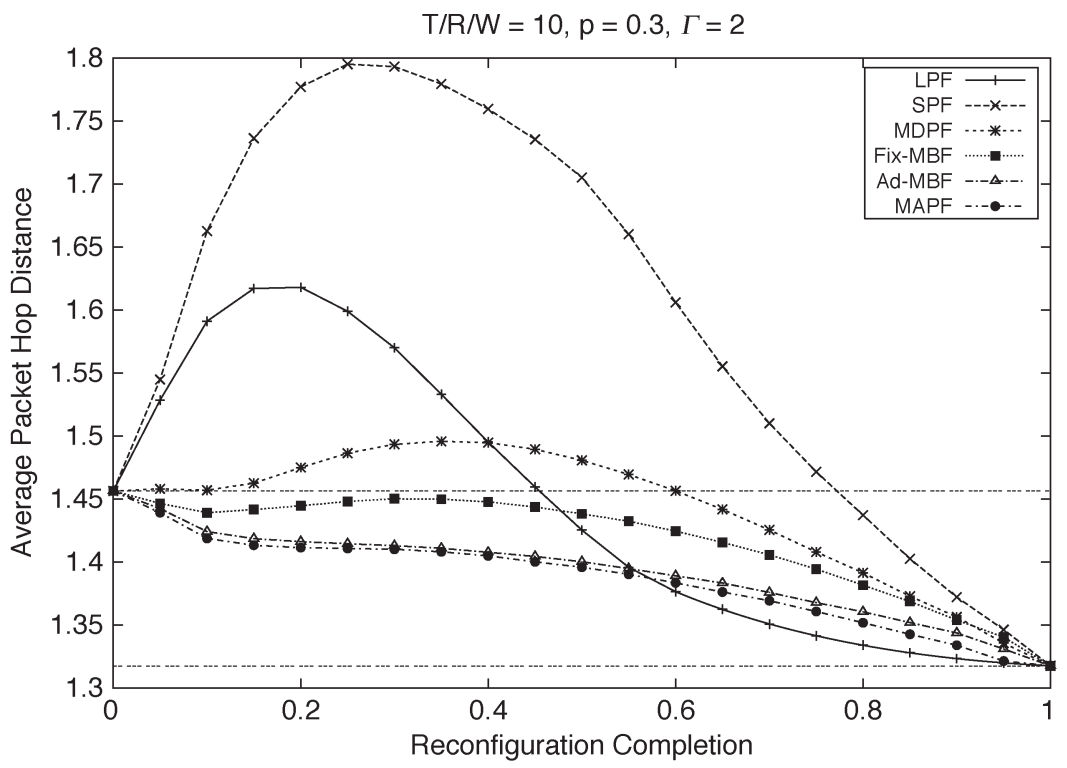

(a)

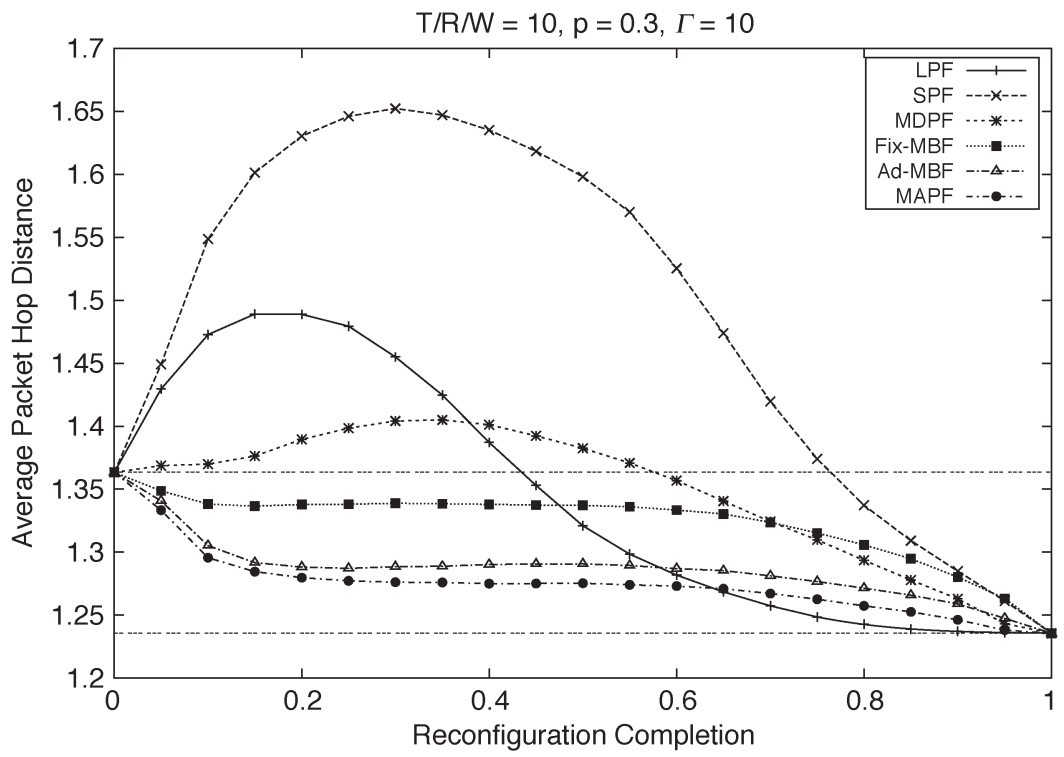

(b)

Fig. 8. Average packet hop distance for $T / R / W=10$ when (a) $p=0.3, \Gamma=2$, and (b) $p=0.3, \Gamma=10$.

packet hop distance $\alpha(T)$. The latter is more important from a user's viewpoint and should be taken with the highest priority in reconfiguration decisions if one would like to provide high quality of service to users. It has been shown that our proposed algorithms show better $\alpha(T)$ than previous algorithms. The Fix-MBF algorithm shows better performance than previous algorithms and with comparable computation time. Furthermore, the Ad-MBF and MAPF algorithms provide the best performance, and their computation time falls into practical domain for a moderate-size network even though it is longer than others.

\section{APPENDIX}

The notation used in this paper is shown as follows. $l_{i}$ $i$ th lightpath in the new logical topology.
$S$

Set of the new lightpaths having conflict relations with the lightpaths in the old topology, i.e., $S=\left\{l_{1}, l_{2}, \ldots, l_{|S|}\right\}$.

$l_{i}^{\prime} \quad i$ th lightpath in the old logical topology.

$S^{\prime} \quad$ Set of the old lightpaths having conflict relations with the lightpaths in the new topology, i.e., $S^{\prime}=\left\{l_{1}^{\prime}, l_{2}^{\prime}, \ldots, l_{\left|S^{\prime}\right|}^{\prime}\right\}$.

$G_{a}\left(V_{a}, E_{a}\right) \quad$ Undirected bipartite auxiliary graph where $V_{a}=S \cup S^{\prime}$ and $E_{a}=\left\{\left(l_{i}, l_{j}^{\prime}\right) \mid\right.$ if $l_{i} \in S$ is in conflict with $\left.l_{j}^{\prime} \in S^{\prime}\right\}$ denote the sets of vertices and edges, respectively.

$T_{N}$

$T_{O}$

$T\left(l_{i}\right)$

Target (new) logical topology.

Current (old) logical topology.

Logical topology in which only new lightpath $l_{i}$ is established over logical topology $T$.

$D_{i} \quad$ Number of disrupted transceivers at stage $i$. 
$C_{i} \quad$ Number of the old lightpaths disrupted at stage $i$. $g\left(l_{i}\right) \quad$ Gain obtained from establishing new lightpath $l_{i} \in S$.

$c\left(l_{i}\right) \quad$ Cost for establishing new lightpath $l_{i} \in S$.

$B\left(l_{i}\right) \quad$ Benefit obtained from establishing a new lightpath $l_{i} \in S$.

$N\left(l_{i}\right) \quad$ Set of old lightpaths having conflict relations with new lightpath $l_{i} \in S$.

$x \quad$ An $(s-d)$ pair; also used to indicate a path between an $(s-d)$ pair.

$X \quad$ Set of all $(s-d)$ pairs.

$X^{\prime} \quad$ Set of $(s-d)$ pairs that have paths passing through new lightpath $l_{i}$ in the new logical topology.

$X^{\prime \prime} \quad$ Set of $(s-d)$ pairs that have paths passing through old lightpaths $l_{i}^{\prime}$ in conflict relation with $l_{i}$.

$W_{T}(x) \quad$ Weighted packet hop distance between an $(s-d)$ pair $x$ under logical topology $T$.

$\alpha(T) \quad$ Average packet hop distance under logical topology $T$.

\section{ACKNOWLEDGMENT}

The authors would like to thank the reviewers for their constructive comments, which greatly helped to improve the paper.

\section{REFERENCES}

[1] R. Ramaswami and K. N. Sivarajan, Optical Networks-A Practical Perspective, 2nd ed. San Francisco, CA: Morgan Kaufmann, 2002.

[2] B. Mukherjee, D. Banerjee, and S. Ramamurthy, "Some principles for designing a wide-area optical network," IEEE/ACM Trans. Netw., vol. 4, no. 5, pp. 684-696, Oct. 1996

[3] A. Banerjee, J. Drake, J. Lang, B. Turner, K. Kompella, and Y. Rekhter, "Generalized multiprotocol label switching: An overview of routing and management enhancements," IEEE Commun. Mag., vol. 39, no. 1, pp. 144-150, Jan. 2001.

[4] A. Banerjee, J. Drake, J. Lang, B. Turner, D. Awduche, L. Berger, K. Kompella, and Y. Movaz, "Generalized multiprotocol label switching: An overview of signaling enhancements and recovery techniques," IEEE Commun. Mag., vol. 39, no. 7, pp. 144-151, Jul. 2001.

[5] J. Lawrence, "Designing multiprotocol label switching networks," IEEE Commun. Mag., vol. 39, no. 7, pp. 134-142, Jul. 2001.

[6] G. N. Rouskas and M. H. Ammar, "Dynamic reconfiguration in multihop WDM networks," J. High Speed Netw., vol. 4, no. 3, pp. 221-238, 1995.

[7] R. Ramaswami and K. N. Sivarjan, "Design of logical topologies for wavelength-routed optical networks," IEEE J. Sel. Areas Commun., vol. 14, no. 5, pp. 840-851, Jun. 1996.

[8] D. Banerjee and B. Mukherjee, "Wavelength-routed optical networks: Linear formulation, resource budgeting tradeoffs, and a reconfiguration study," IEEE/ACM Trans. Netw., vol. 8, no. 5, pp. 598-607, Oct. 2000.

[9] M. Kato and Y. Oie, "Reconfiguration procedures for torus lightwave networks," in Proc. IEEE Int. Conf. Communications (ICC), Atlanta, GA, Jun. 1998, pp. 531-536.

[10] E. Modiano and A. Narula-Tam, "Survivable routing of logical topologies in WDM networks," in Proc. IEEE INFOCOM, Anchorage, AK, 2001, pp. 348-357.

[11] N. Sreenath, C. Siva Ram Murthy, B. H. Gurucharan, and G. Mohan, "A two-stage approach for virtual topology reconfiguration of WDM optical networks," Opt. Netw. Mag., vol. 2, no. 3, pp. 58-71, May/Jun. 2001.

[12] H. Takagi, Y. Zhang, X. Jia, and H. Takagi, "Reconfiguration heuristics for logical topologies in wide-area WDM networks," in Proc. IEEE Global Telecommunications (GLOBECOM), Taipei, Taiwan, Nov. 2002, pp. 2701-2705.

[13] I. Chlamtac, A. Ganz, and G. Karmi, "Lightpath communications: An approach to high bandwidth optical WAN's," IEEE Trans. Commun., vol. 40, no. 7, pp. 1171-1182, Jul. 1992.
[14] B. Ramamurthy and A. Ramakrishnan, "Virtual topology reconfiguration of wavelength-routed optical WDM networks," in Proc. IEEE Global Telecommunications (GLOBECOM), San Francisco, CA, Nov. 2000, pp. $1269-1275$.

[15] C. S. R. Murthy and M. Gurusamy, WDM Optical Networks. Englewood Cliffs, NJ: Prentice-Hall, 2002.

[16] K. Lee and K. Y. Siu, "On the reconfigurability of single-hub WDM ring networks," IEEE/ACM Trans. Netw., vol. 11, no. 2, pp. 273-284, Apr. 2003.

[17] A. Gencata and B. Mukherjee, "Virtual-topology adaptation for WDM mesh networks under dynamic traffic," IEEE/ACM Trans. Netw., vol. 11, no. 2, pp. 236-247, Apr. 2003.

[18] J. F. P. Labourdette, G. W. Hart, and A. S. Acampora, "Branch-exchange sequences for reconfiguration of lightwave networks," IEEE Trans. Commun., vol. 42, no. 10, pp. 2822-2832, Oct. 1994.

[19] A. Narula-Tam and E. Modiano, "Dynamic load balancing in WDM packet networks with and without wavelength constraints," IEEE $J$. Sel. Areas Commun., vol. 18, no. 10, pp. 1972-1979, Oct. 2000.

[20] I. Baldine and G. N. Rouskas, "Traffic adaptive WDM networks: A study of reconfiguration issues," J. Lightw. Technol., vol. 19, no. 4, pp. 433-455, Apr. 2001.

[21] W. Golab and R. Boutaba, "Policy-driven automated reconfiguration for performance management in WDM optical networks," IEEE Commun. Mag., vol. 42, no. 1, pp. 44-51, Jan. 2004.

[22] A. Narula-Tam and E. Modiano, "Dynamic load balancing for WDMbased packet networks," in Proc. IEEE INFOCOM, Tel Aviv, Israel, Mar. 2000, pp. 1010-1019.

[23] G. Mohan, P. H. H. Ernest, and V. Bharadwaj, "Virtual topology reconfiguration in IP/WDM optical ring networks," Comput. Commun., vol. 26, no. 2, pp. 91-102, Feb. 2003

[24] K. Bala, G. Ellinas, and M. Post, "Towards hitless reconfiguration in WDM optical networks for ATM transport," in Proc. IEEE Global Telecommunications (GLOBECOM), London, U.K., 1996, pp. 316-320.

[25] G. S. K. Reddy, C. S. R. Murthy, and G. Manimaran, "Reconfiguration based failure restoration in wavelength-routed WDM networks," in Proc. IEEE Dependable Systems and Networks, New York, Jun. 2000, pp. 543-552.

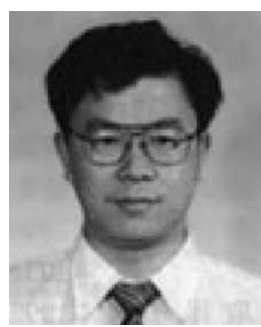

Yongbing Zhang (M'96) received the B.S. degree in electrical engineering from the Polytechnical University, Kanagawa, Japan, in 1984, and the M.S. and Dr.Eng. degrees in computer science both from the University of Electro-Communications, Tokyo, Japan, in 1989 and 1992, respectively.

From 1992 to 1996, he was a Research Associate with the Department of Computer Science at the University of Electro-Communications. In 1996, he joined the Institute of Policy and Planning Sciences, University of Tsukuba, Tsukuba, Ibaraki, Japan. $\mathrm{He}$ is now an Associate Professor at the Graduate School of Systems and Information Engineering of the University of Tsukuba. His research interests include distributed/parallel computer systems, communication networks, and performance evaluation.

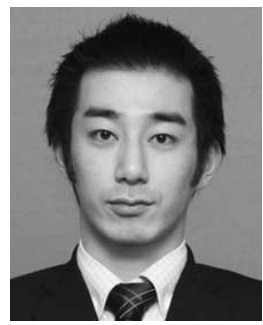

Masaki Murata received the B.S. degree in policy and planning sciences and the M.S. degree in management science from the University of Tsukuba, Tsukuba, Ibaraki, Japan, in 2000 and 2004, respectively.

He is now a Software Engineer with NTT Internet, Inc., Tokyo, Japan. 


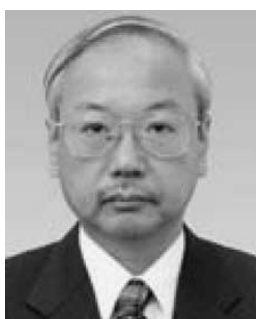

Hideaki Takagi (S'80-M'83-SM'87-F'96) received the B.S. and M.S. degrees in physics from the University of Tokyo, Tokyo, Japan, in 1972 and 1974, respectively, and the Ph.D. degree in computer science from the University of California, Los Angeles, in 1983.

Prior to the current position, he was a Consultant Researcher at IBM Research, Tokyo Research Laboratory, Professor and Chair at the Institute of Policy and Planning Sciences and Vice President of the University of Tsukuba, Tsukuba, Ibaraki, Japan. He is currently a Professor at the Graduate School of Systems and Information Engineering of the University of Tsukuba. His research interest includes enumerative combinatorics, probability models, and performance evaluation of computer and communication systems.

Dr. Takagi is an International Federation for Information Processing (IFIP) Silver Core holder.

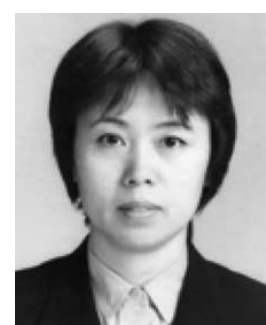

Yusheng Ji (M'94) received the B.E., M.E., and D.E. degrees in electrical engineering from the University of Tokyo, Tokyo, Japan, in 1984, 1986, and 1989, respectively.

She joined the National Center for Science Information Systems in 1990. Currently, she is an Associate Professor at the National Institute of Informatics, Tokyo, Japan, and the Graduate University for Advanced Studies, Kanagawa, Japan. Her research interests include network architecture, traffic control, and performance analysis for quality of service provisioning in high-speed networks.

Dr. Ji is a Member of Information Processing Society of Japan (IPSJ) and the Institute of Electronics, Information and Communication Engineers (IEICE). 